\title{
Pancreatic adipose tissue infiltration, parenchymal steatosis and beta cell function in humans
}

\author{
Paul Begovatz • Chrysi Koliaki • Katharina Weber • \\ Klaus Strassburger • Bettina Nowotny • Peter Nowotny • \\ Karsten Müssig • Jürgen Bunke • Giovanni Pacini • \\ Julia Szendrödi • Michael Roden
}

Received: 14 October 2014 / Accepted: 4 February 2015 / Published online: 5 March 2015

(C) Springer-Verlag Berlin Heidelberg 2015

\begin{abstract}
Aims/hypothesis This study aimed to perform a comprehensive analysis of interlobular, intralobular and parenchymal pancreatic fat in order to assess their respective effects on beta cell function.

Methods Fifty-six participants (normal glucose tolerance [NGT] ( $n=28)$, impaired fasting glucose (IFG) and/or impaired glucose tolerance (IGT) $(n=14)$ and patients with type 2 diabetes $(n=14))$ underwent a frequent-sampling OGTT and non-invasive magnetic resonance imaging (MRI; whole-body and pancreatic) and proton magnetic resonance spectroscopy ( ${ }^{1} \mathrm{H}-\mathrm{MRS}$; liver and pancreatic fat). Total pancreatic fat was assessed by a standard $2 \mathrm{~cm}^{3}{ }^{1} \mathrm{H}-\mathrm{MRS}$ method, intralobular fat by $1 \mathrm{~cm}^{3}{ }^{1} \mathrm{H}$-MRS that avoided interlobular fat within
\end{abstract}

P. Begovatz $\cdot$ C. Koliaki $\cdot$ K. Weber $\cdot$ B. Nowotny $\cdot$ P. Nowotny $\cdot$ K. Müssig $\cdot$ J. Szendrödi $\cdot$ M. Roden $(\bowtie)$

Institute for Clinical Diabetology, German Diabetes Center, Leibniz Center for Diabetes Research at Heinrich Heine University, Auf $\mathrm{m}$ Hennekamp 65, 40225 Düsseldorf, Germany

e-mail: michael.roden@ddz.uni-duesseldorf.de

P. Begovatz $\cdot$ C. Koliaki $\cdot$ K. Weber $\cdot$ B. Nowotny $\cdot$ P. Nowotny $\cdot$

K. Müssig $\cdot$ J. Szendrödi $\cdot$ M. Roden

German Center for Diabetes Research, Düsseldorf, Germany

K. Strassburger

Institute for Biometry and Epidemiology, German Diabetes Center,

Düsseldorf, Germany

B. Nowotny $\cdot \mathrm{K}$. Müssig $\cdot J$. Szendrödi $\cdot$ M. Roden

Department of Endocrinology and Diabetology, Medical Faculty,

Heinrich Heine University Düsseldorf, Düsseldorf, Germany

J. Bunke

Philips Healthcare, Hamburg, Germany

G. Pacini

Metabolic Unit, Institute of Biomedical Engineering (ISIB-CNR),

Padua, Italy modified DIXON (mDIXON) water images, and parenchymal fat by a validated mDIXON-MRI fat-fraction method.

Results Comparison of ${ }^{1} \mathrm{H}-\mathrm{MRS}$ techniques revealed an inhomogeneous distribution of interlobular and intralobular adipose tissue, which increased with decreasing glucose tolerance. mDIXON-MRI measurements provided evidence against uniform steatosis, revealing regions of parenchymal tissue void of lipid accumulation in all participants. Total $(r=0.385, p<0.01)$ and intralobular pancreas adipose tissue infiltration $(r=0.310, p<0.05)$ positively associated with age, but not with fasting or $2 \mathrm{~h}$ glucose levels, BMI or visceral fat content (all $p>0.5$ ). Furthermore, no associations were found between total and intralobular pancreatic adipose tissue infiltration and insulin secretion or beta cell function within NGT, IFG/IGT or patients with type 2 diabetes (all $p>0.2$ ). Conclusions/interpretation The pancreas does not appear to be another target organ for abnormal endocrine function because of ectopic parenchymal fat storage. No relationship was found between pancreatic adipose tissue infiltration and beta cell function, regardless of glucose tolerance status.

Keywords Beta cell · Insulin · Insulin sensitivity · Lipids · Magnetic resonance imaging $\cdot$ Pancreas

\begin{tabular}{ll}
\multicolumn{2}{l}{ Abbreviations } \\
${ }^{1} \mathrm{H}-\mathrm{MRS}$ & Proton magnetic resonance spectroscopy \\
AUC $_{\mathrm{CP}}$ & AUC for C-peptide \\
AUC $_{\mathrm{GLU}}$ & AUC for glucose \\
$\mathrm{AUC}_{\mathrm{INS}}$ & AUC for insulin \\
$\mathrm{HCL}$ & Hepatic lipid content \\
IFG & Impaired fasting glucose \\
IGT & Impaired glucose tolerance \\
mDIXON & Modified DIXON fat/water imaging sequence \\
MRI & Magnetic resonance imaging \\
NGT & Normal glucose tolerance
\end{tabular}




$\begin{array}{ll}\text { OGIS } & \text { Oral glucose insulin sensitivity index } \\ \text { ROI } & \text { Region of interest } \\ \text { STEAM } & \text { Stimulated echo acquisition mode } \\ \text { TSE } & \text { Turbo-spin-echo } \\ \text { VAPOR } & \text { Variable power and optimization relaxation } \\ \text { VAT } & \text { Visceral adipose tissue }\end{array}$

\section{Introduction}

Type 2 diabetes generally develops when beta cells fail to maintain adequate insulin secretion for the corresponding insulin resistance, because of loss of function or apoptosis [1]. Dysfunction of beta cells occurs in islets susceptible to inherited abnormalities of function and insulin secretion, as opposed to islets capable of producing an adaptive response to insulin resistance [2]. Possible underlying mechanisms leading to beta cell dysfunction are abnormal mitochondrial function and oxidative stress, mainly due to glucose toxicity $[3,4]$ and lipotoxicity, along with elevated concentrations of NEFA [5, 6].

Lipotoxicity results from augmented adipose tissue lipolysis leading to triacylglycerol overflow and ectopic accumulation of lipid metabolites in non-adipose tissues. Previous research has focused mainly on the role of increased lipid accumulation in the liver [7] and skeletal muscle [8-10] for the development of insulin resistance and type 2 diabetes. However, recent data also show that obesity leads to an increase of pancreatic fat in animals [11-13] and humans [14-16], with evidence of triacylglycerol overflow inducing pancreatic steatosis and beta cell dysfunction [17-20]. Proton magnetic resonance spectroscopy $\left({ }^{1} \mathrm{H}-\mathrm{MRS}\right)$ is the current gold standard for non-invasive in vivo quantification of triacylglycerol content in liver and skeletal muscle, and has been validated against histological and biochemical quantification in human biopsies [7]. More recently, ${ }^{1} \mathrm{H}-\mathrm{MRS}$ [17-19] and magnetic resonance imaging (MRI) by fat/water imaging techniques $[16,21-23]$ have also been applied to determine pancreatic fat. These studies aimed not only to quantify the fat content of the pancreas, but also to examine the possible interaction of pancreatic fat with beta cell function and insulin secretion [18-20].

However, conflicting results were reported both with regard to the amount of pancreatic fat $[16,17,19,21]$ and the association with beta cell function in humans [18-21, 24]. These differences could be attributed to the various fat compartments found within the pancreas, and the variability they cause in invasive and non-invasive measurements. Similar to visceral adipose fat (VAT), peripancreatic, interlobular and intralobular adipose tissue $[11,14,15]$ may alter beta cell function through the release of adipocytokines $[6,25]$. On the other hand, lipid accumulation within exocrine or endocrine cells [12], or specifically in beta cells, could result in parenchymal or intracellular pancreatic steatosis [26, 27], activating cellular mechanisms in analogy to hepatic steatosis. Distinguishing between these compartments in vivo could therefore aid in the accurate assessment of the specific effects of individual fat depots on beta cell function.

Thus, this study aimed to apply different ${ }^{1} \mathrm{H}$-MRS and MRI techniques to non-invasively quantify pancreatic fat compartments in participants with normal glucose tolerance (NGT), impaired fasting glucose (IFG) and/or impaired glucose tolerance (IGT), and in patients with type 2 diabetes. Total pancreatic fat content was measured with a standard ${ }^{1} \mathrm{H}-\mathrm{MRS}$ technique $[17,19]$, intralobular fat content by a ${ }^{1} \mathrm{H}-\mathrm{MRS}$ method with localization via modified DIXON (mDIXON) [28] water images to avoid interlobular fat [28] and, finally, parenchymal fat accumulation was quantified by a validated [29] dual-echo mDIXON-MRI method. These measures of pancreatic fat compartments were then tested for correlation with beta cell function [30] derived from a frequent-sampling OGTT, as well as with VAT and hepatocellular fat content (HCL).

\section{Methods}

Participants The study population comprised 56 white participants, 28 with NGT, 14 with IFG/IGT and 14 patients with type 2 diabetes of $5.8 \pm 4.9$ years (mean $\pm \mathrm{SD}$ ) known duration. Participants were excluded in the case of a medical history of pancreatitis, and/or alcohol consumption greater than $20 \mathrm{~g}$ per day for men or $10 \mathrm{~g}$ per day for women. Participants were also excluded if receiving treatment with thiazolidinediones, incretin mimetics or insulin, which interfere with ectopic fat deposition or beta cell function. The ${ }^{1} \mathrm{H}-\mathrm{MRS} / \mathrm{MRI}$ protocol and OGTT were performed within 7 days, and patients withdrew from their oral glucose-lowering medication for at least 3 days before the metabolic test, as reported previously [31]. Type 2 diabetes patients were treated with metformin only $(n=6)$, metformin plus dipeptidyldipeptidase 4 (DPP4) inhibitor $(n=2)$, or sulfonylurea plus DPP4 inhibitor $(n=2)$. Four patients were using statin treatment. Finally, all participants gave written informed consent before inclusion in the study, which was approved by the institutional review ethics board and conducted in accordance with the Declaration of Helsinki.

OGTT A 2 h OGTT (75 g) was performed after 3 days of carbohydrate-enriched diet and overnight fast. Blood was taken at 0,30,60 and $120 \mathrm{~min}$ for measuring glucose, insulin and C-peptide levels. Plasma glucose concentration was quantified with the EKF Biosen C-Line glucose analyser (EKF Diagnostic, Barleben, Germany) using the glucose oxidase method; serum insulin and C-peptide were measured by radioimmunoassay (Millipore, St Charles, MO, USA). The 
AUC was measured using the trapezoidal rule. The AUC of glucose $\left(\mathrm{AUC}_{\mathrm{GLUC}}\right)$ was used to quantify glucose tolerance on intervention. Insulin sensitivity was assessed from the oral glucose insulin sensitivity index (OGIS) [30, 32], which provides a measurement of insulin-mediated glucose clearance. Insulin secretion was assessed through C-peptide AUC $\left(\mathrm{AUC}_{\mathrm{CP}}\right)$, and the post-hepatic peripheral (systemic) appearance of insulin with $\mathrm{AUC}_{\mathrm{INS}}$ for the $2 \mathrm{~h}$ test. The interplay between insulin sensitivity and hormone secretion that describes the beta cell adaptive response to changes of insulin resistance [33] was determined by the adaptation index $\left(\mathrm{OGIS} \times \mathrm{AUC}_{\mathrm{CP}}\right)$. This variable was originally developed for the intravenous glucose test, but was validated as a reliable determinant of beta cell function derived from the OGTT [34, 35]. Finally, beta cell function, defined as hormone secretion relative to glucose stimulation, was derived from the ratios of total C-peptide to glucose $\left(\mathrm{AUC}_{\mathrm{CP}} / \mathrm{AUC}_{\mathrm{GLUC}}\right)$, which is defined here as the insulinogenic index [30, 36, 37].

MRS and MRI All measurements were performed using a whole-body 3.0-T Achieva scanner (Philips Healthcare, Best, the Netherlands). VAT was determined from the abdominal images from a T1-weighted turbo-spin-echo (TSE) sequence with $10 \mathrm{~mm}$ slice thickness [38]. Participants were then repositioned for the MRI/MRS protocol for HCL and pancreatic fat conducted with a 16 channel XL torso phased-array receiver coil (Philips Healthcare). HCL and pancreatic fat were determined by ${ }^{1} \mathrm{H}-\mathrm{MRS}$ (single voxel stimulated echo acquisition mode [STEAM], repetition time $4 \mathrm{~s}$, echo time $10 \mathrm{~ms}$, scans 32). Expiration respiratory triggering was used to avoid motion artifacts and VAT from outside the pancreas $[19,23]$. The entire protocol was conducted within $45 \mathrm{~min}$. ${ }^{1}$ H-MRS data were analysed with the software package NUTS (Acorn NMR, Freemont, CA, USA) and fat fraction was expressed as [fat/(water + fat $)]$.

Total pancreatic fat Standard measurements of ${ }^{1} \mathrm{H}-\mathrm{MRS}$ pancreatic fat served as a control, and were performed with a $(20 \times$ $10 \times 10 \mathrm{~mm}^{3}$ ) voxel, with localization via navigator-triggered clinical T2-weighted TSE images $(2,081 \mathrm{~ms}$ repetition time, $80 \mathrm{~ms}$ echo time, $4 \mathrm{~mm}$ slice thickness). VAT outside of the pancreas was avoided throughout all slices in coronal and transverse planes. The clinical T2-weighted images were not able to distinguish between adipose tissue infiltration and parenchymal tissue. Thus, results yielded a measure of total pancreatic fat consisting of peripancreatic, interlobular and intralobular adipose tissue infiltration and parenchymal fat.

Intralobular pancreatic fat Transverse and coronal dual-echo mDIXON abdominal fat/water images were then acquired in separate $19 \mathrm{~s}$ breathholds, via a three-dimensional $\mathrm{T} 1$ fast field gradient echo pulse sequence $\left(2 \mathrm{~mm}\right.$ isotropic resolution, $5^{\circ}$ flip angle, $5.0 \mathrm{~ms}$ repetition time, $1.2 \mathrm{~ms}$ echo time, $2.5 \mathrm{~ms}$ echo time) [29]. Single voxel $\left(10 \times 10 \times 10 \mathrm{~mm}^{3}\right){ }^{1} \mathrm{H}-\mathrm{MRS}$ was performed with voxel localization in the body or tail of the pancreas via the high-resolution transverse and coronal mDIXON water images. Measurements were conducted immediately after the mDIXON imaging to avoid the volunteer's movement between pancreatic fat measurements. The voxel was placed not only to avoid VAT outside of the pancreas, but also regions of pure peripancreatic and interlobular fat. Thus, this method provided a measure of intralobular adipose tissue infiltration including parenchymal fat content. In all ${ }^{1} \mathrm{H}$-MRS pancreatic fat measurements, fat was calculated from the methylene signal at $1.3 \mathrm{ppm}$.

Parenchymal pancreas fat The low-fat PRIDE software package (Philips Healthcare) was used in post-acquisition to reconstruct pixel by pixel fat-fraction maps from the mDIXON fat/ water images. Fat-fraction measurements via mDIXON fat/ water imaging with the low-fat PRIDE algorithm were previously validated against ${ }^{1} \mathrm{H}$-MRS in vivo HCL measurements [29], and found to have a CV of $4.3 \%$. Measurements were performed after patient examination with the placement of $100 \mathrm{~mm}^{2}$ regions of interest (ROIs), which were defined to encompass the anterior and posterior walls of the pancreas in the transverse plane. Parenchymal fat measurements were represented as the average of four ROIs that avoided all regions of visible adipose tissue infiltration. Thus, this method yielded a measure of uniform pancreatic steatosis within parenchymal tissue.

${ }^{1} H$-MRS of liver fat Single voxel $\left(30 \times 30 \times 20 \mathrm{~mm}^{3}\right){ }^{1} \mathrm{H}-\mathrm{MRS}$ was used to measure liver fat using the above described STEAM sequence to assess water content and a variable power and optimization relaxation (VAPOR) water-suppressed STEAM sequence. Fat was expressed as the summation of the VAPOR water-suppressed fat peaks of methylene (1.3 ppm) and methyl $(0.9 \mathrm{ppm})$. Measurements were T2 corrected to obtain values of proton density fat fraction, and corrected for per cent of total hepatic fat content [39].

Validation of ${ }^{1} H$-MRS pancreas and liver fat measurements The mean intra-individual $\mathrm{CV}$ between eight repeated hepatic and pancreatic measurements was conducted from the respective ${ }^{1} \mathrm{H}-\mathrm{MRS}$ measurements used in the present study. The CVs and Pearson correlation coefficient $(r)$ were as follows $(\mathrm{CV}, r)$ : ${ }^{1} \mathrm{H}$-MRS of liver fat $(7.5 \%, 0.99)$, total pancreatic fat $(7.5 \%, 0.95)$ and parenchymal pancreatic fat $(9.5 \%, 0.94)$.

Statistical analysis Results were expressed as mean $\pm \mathrm{SD}$ or median (first quartile, third quartile) for cases of skewed distribution. Differences between groups were assessed using Wilcoxon-Mann-Whitney-tests, while differences between methods were assessed with a Wilcoxon signed-rank test. 
Associations of pancreatic fat and markers for obesity with ectopic fat distribution and beta cell function were assessed using Pearson correlations adjusted for age, sex and insulin sensitivity. Graphs were created using GraphPad PRISM V6 (GraphPad Software, La Jolla, CA, USA). All statistical analyses were performed using SAS for Windows Version 9.2 (SAS Institute, Cary, NC, USA). A $p$ value $\leq 0.05$ was considered to indicate a statistically significant difference.

\section{Results}

Participant characteristics Patients with type 2 diabetes were older and had increased $\mathrm{HbA}_{1 \mathrm{c}}$ compared with those with NGT and IFG/IGT, and had increased BMI and HCL compared with participants with NGT (Table 1). There were no differences in VAT and waist circumference between all participants. IFG/IGT participants had higher levels of fasting and $2 \mathrm{~h}$ glucose, increased insulin secretion (Fig. 1) and decreased insulin sensitivity and beta cell function compared with NGT. Furthermore, patients with type 2 diabetes demonstrated increased fasting and $2 \mathrm{~h}$ glucose levels compared with participants with NGT or IFG/IGT, along with decreased insulin sensitivity and beta cell function. However, $2 \mathrm{~h}$ insulin secretion results in participants with diabetes were similar to those of participants with NGT.
Pancreatic fat content Clinical T2-weighted imaging showed that the pancreas was clearly identifiable in NGT participants with low VAT (Fig. 2a-d). However, distinction of pancreatic parenchymal tissue from VAT for ${ }^{1} \mathrm{H}$-MRS localization was more difficult in participants with IFG/IGT or diabetes, due in part to irregularities in both the organ shape and size. The total pancreatic fat content was slightly lower in participants with NGT $(1.95 \%[0.29 \%, 6.35 \%])$ than in those with IFG/IGT $(4.79 \%[1.55 \%, 8.27 \%])$, in line with BMI and VAT measurements in the two groups. However, total pancreatic fat content and the variability of fat measurements were higher $(p<0.05)$ in patients with diabetes $(8.35 \%[5.64 \%, 13.10 \%])$.

The high-resolution mDIXON water images clearly separated interlobular fat from parenchymal tissue, and accurately displayed the irregular shape of the pancreas in participants with IFG/IGT or type 2 diabetes (Fig. 2e-h). Within the pancreases of participants with NGT or IFG/IGT mDIXON water imaging often did not reveal any regions of pancreatic adipose tissue infiltration. However, in other cases, regions of pure adipose tissue infiltration were visible within the pancreas, and dissected large regions of parenchymal pancreatic tissue. Compared with total pancreatic fat measurements $(p<0.05)$, intralobular ${ }^{1} \mathrm{H}-\mathrm{MRS}$ measurements that avoided these regions of interlobular fat led to lower pancreatic fat values in participants with NGT $(1.49 \%[0.01 \%, 2.30 \%])$ and in those with IFG/IGT $(0.67 \%$ [0.00, 5.07\%]). Furthermore, mDIXON imaging within patients with type 2 diabetes found that increased adipose tissue infiltration led to

Table 1 The anthropometric characteristics of participants

\begin{tabular}{|c|c|c|c|}
\hline Characteristic & NGT & IFG/IGT & Type 2 diabetes \\
\hline$n$ & 28 & 14 & 14 \\
\hline IFG, IGT, IFG/IGT & - & $5,3,6$ & - \\
\hline Men/women & $13 / 15$ & $8 / 6$ & $11 / 3$ \\
\hline Age (years) & $49.7 \pm 11.7$ & $50.9 \pm 10.8$ & $61.1 \pm 4.5^{* \dagger}$ \\
\hline Waist circumference $(\mathrm{cm})$ & $101.0 \pm 8.5$ & $106.9 \pm 13.1$ & $105.1 \pm 9.3$ \\
\hline BMI $\left(\mathrm{kg} / \mathrm{m}^{2}\right)$ & $26.3 \pm 4.5$ & $29.2 \pm 5.1$ & $30.2 \pm 5.3^{*}$ \\
\hline $\mathrm{HbA}_{1 \mathrm{c}}(\%)$ & $5.5 \pm 0.6$ & $5.6 \pm 0.4$ & $8.1 \pm 1.5^{* *+\dagger}$ \\
\hline $\mathrm{HbA}_{1 \mathrm{c}}(\mathrm{mmol} / \mathrm{mol})$ & $35.6 \pm 3.2$ & $37.6 \pm 4.6$ & $64.6 \pm 16.6^{* *+\dagger}$ \\
\hline Fasting glucose (mmol/l) & $4.2 \pm 0.4$ & $5.7 \pm 0.8^{*}$ & $9.5 \pm 2.2^{* * \dagger \dagger}$ \\
\hline Glucose $120 \mathrm{~min}(\mathrm{mmol} / \mathrm{l})$ & $4.6 \pm 1.5$ & $8.2 \pm 1.6^{*}$ & $18.7 \pm 3.6^{* *+\dagger}$ \\
\hline VAT (ml) & $2,503(1,701,4,237)$ & $3,101(1,811,5,100)$ & $3,076(1,541,3,708)$ \\
\hline HCL $(\%)$ & $2.10(0.38,3.53)$ & $5.95(0.53,8.89)$ & $8.00(5.38,11.83)^{*}$ \\
\hline OGIS & $470 \pm 93$ & $350 \pm 141^{*}$ & $209 \pm 56^{* * \dagger}$ \\
\hline $\mathrm{AUC}_{\mathrm{INS}} 120 \mathrm{~min}(\mathrm{nmol} / \mathrm{l})$ & $28.1(20.8,47.6)$ & $49.4(36.4,79.2)^{*}$ & $22.3(16.3,33.7)^{\dagger}$ \\
\hline Adaptation index & $0.40 \pm 0.11$ & $0.30 \pm 0.10^{*}$ & $0.16 \pm 0.08^{* * \dagger}$ \\
\hline $\begin{array}{l}\mathrm{AUC}_{\mathrm{CP}} / \mathrm{AUC}_{\mathrm{GLUC}} 120 \mathrm{~min} \\
\quad \text { (nmol C-peptide/mmol glucose) }\end{array}$ & $1,021(766,1,539)$ & $570(337,896)^{*}$ & $158(90,180)^{* * \dagger \dagger}$ \\
\hline
\end{tabular}

Data are represented as mean $\pm \mathrm{SEM}$, and median (first quartile, third quartile) in cases of skewed distribution

${ }^{*} p<0.05$ and ${ }^{* *} p<0.001$ vs NGT, ${ }^{\dagger} p<0.05$ and ${ }^{\dagger \dagger} p<0.001$ vs IFG/IGT (Wilcoxon-Mann-Whitney tests) 
Fig. 1 Circulating concentrations of (a) glucose, (b) insulin and (c) C-peptide during a $75 \mathrm{~g}$ OGTT. Data presented as mean \pm SEM. Circles, NGT; squares, IFG/IGT; and triangles, type 2 diabetes
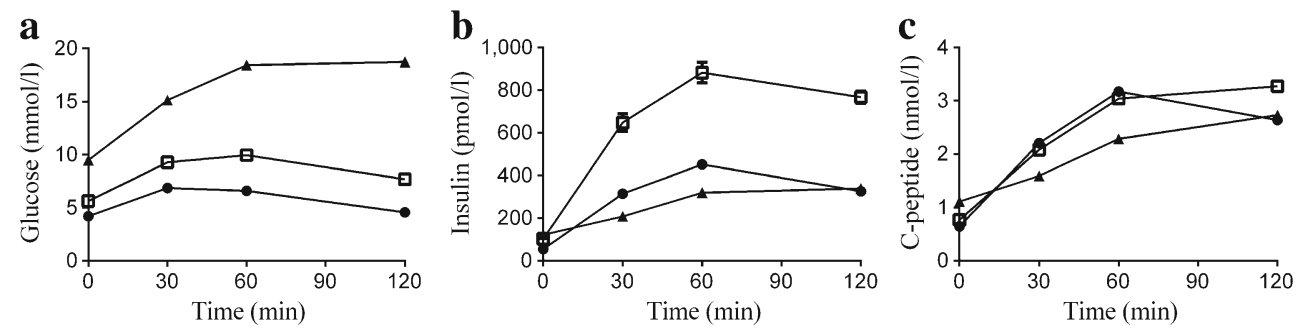

difficulties in distinguishing between regions of intralobular or interlobular fat, due to either marked adipocyte infiltration or organ atrophy. This led to higher intralobular fat measurements in diabetic patients $(6.20 \%$ [5.03\%, 9.47\%]) compared with participants with NGT $(p<0.001)$ or IFG/IGT $(p<0.05)$, in line with total pancreatic fat measurements.

Parenchymal pancreatic fat measurements revealed multiple regions of parenchymal tissue void of lipid accumulation in all participants, thereby providing evidence against uniform pancreatic steatosis (Fig. 2i-1). Parenchymal fat content in participants with NGT $(0.14 \%[-0.10 \%, 0.36 \%])$ or IFG/IGT $(0.49 \%$ $[0.14 \%, 0.88 \%])$ was below the $2 \%$ sensitivity threshold of the fat quantification method, and were in line with intralobular fat measurements. However, the placement of ROIs during post- acquisition allowed for all regions of adipose tissue infiltration to be avoided, and also found no evidence of uniform parenchymal fat accumulation even in patients with type 2 diabetes $(0.37 \%[-0.30 \%, 0.65 \%])$. Further evidence against uniform pancreatic steatosis was found from the analysis of all ${ }^{1} \mathrm{H}-$ MRS spectra obtained from total pancreatic and intralobular fat measurements. In all cases, the spectra exhibited a single broad fat peak in the region of $1.3 \mathrm{ppm}$ that was signature of lipid accumulation within small volumes of pure adipose tissue, rather than the typical distribution of fat peaks obtained in intracellular HCL measurements (Fig. 3).

Fat distribution, insulin sensitivity and beta cell function Prior to adjustments, total pancreatic fat $(r=0.477$,
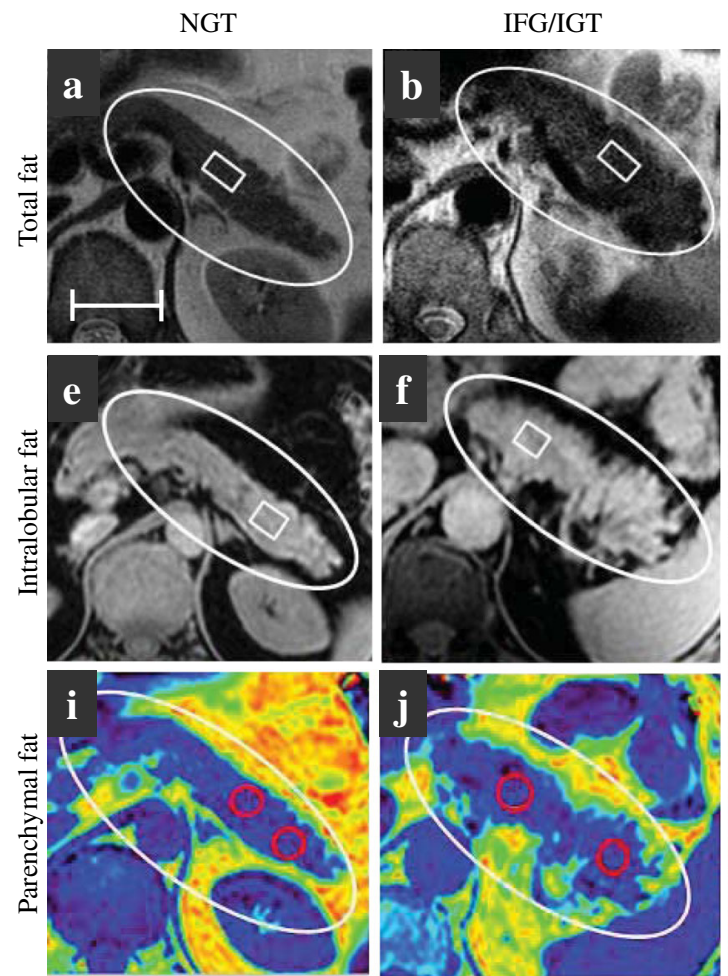

Fig. 2 Examples of pancreatic fat measurements and changes in pancreas (white circles) morphology found within the same NGT (a, e, i), IFG/IGT (b, f, j), and type 2 diabetes (c, $\mathbf{g}, \mathbf{k})$ participant. (a-d) Total pancreatic fat measurements with $2 \mathrm{~cm}^{3}{ }^{1} \mathrm{H}-\mathrm{MRS}$ voxel (white square) localization within clinical T2-weighted images (scale bar, $40 \mathrm{~mm}$ ). (e-h) Intralobular pancreatic fat measurements with $1 \mathrm{~cm}^{3}$ voxel (white square) localization that avoided regions of peripancreatic and/or interlobular fat (black) visible in mDIXON water images. (i-l) Parenchymal fat measurements
Type 2 diabetes
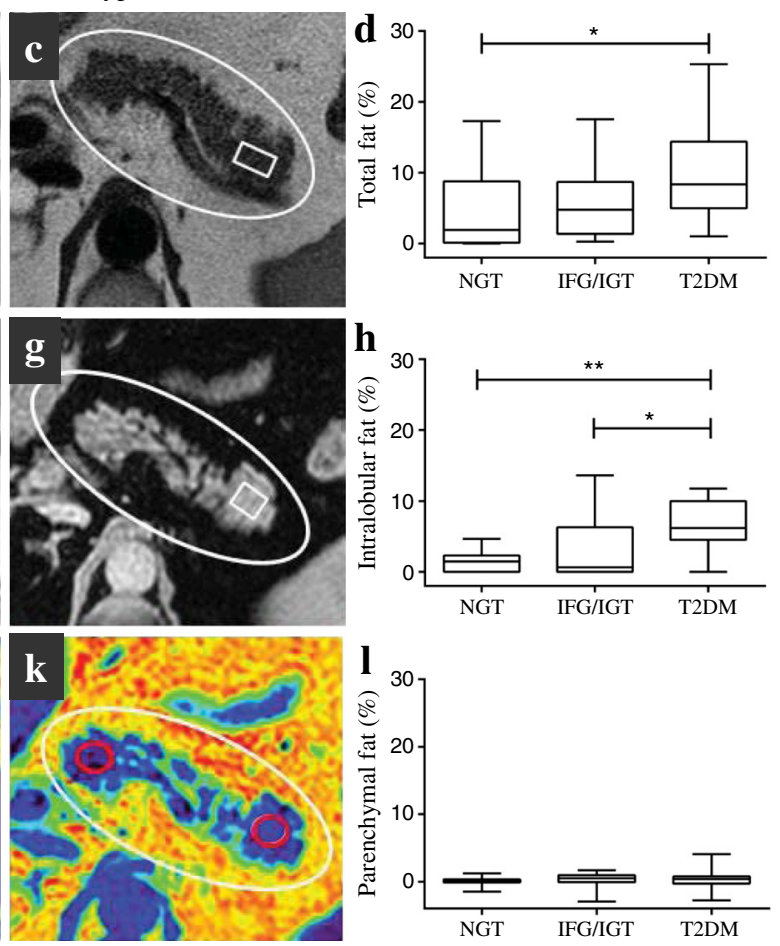

within fat-fraction maps reconstructed from $\mathrm{mDIXON}$ fat/water images to test for uniform pancreatic steatosis. Fat measurements range from $0 \%$ (blue) to $100 \%$ (red), with $100 \mathrm{~mm}^{2}$ ROIs (red circles). Box plots represent the median values of pancreatic fat, upper and lower limits represent the 25th and 75th percentiles, and whiskers the minimum and maximum. ${ }^{*} p<0.05$ and ${ }^{* *} p<0.001$ (Wilcoxon-Mann-Whitney tests). T2DM, type 2 diabetes 

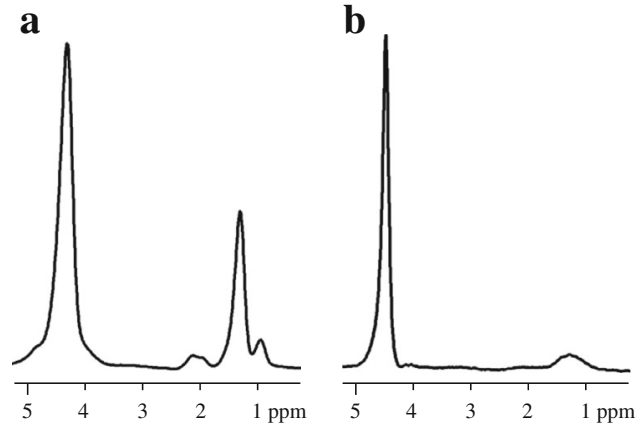

Fig. 3 Comparison of ${ }^{1}$ H-MRS non-water-suppressed HCL and pancreatic fat spectra: (a) $20 \% \mathrm{HCL}$ with clearly defined narrow fat peaks at $2.1 \mathrm{ppm}, 1.3 \mathrm{ppm}$ and $0.9 \mathrm{ppm}$; (b) $14 \%$ total pancreatic fat with single

$p<0.001)$, intralobular fat $(r=0.428, p<0.001)$, and VAT $3(r=0.282, p<0.001)$ positively associated with age. On adjusting for sex and insulin sensitivity, total pancreatic fat $(r=0.385, p<0.01)$ and intralobular fat $(r=0.310, p<0.05)$ associated with age, but not VAT.

After adjusting for age, sex and insulin sensitivity, total pancreatic fat was positively associated with waist circumference $(r=0.326, p<0.05)$, and intralobular fat was associated with HCL $(r=0.346, p<0.05)$. However, no other associations were found between total, intralobular or parenchymal fat and fasting or $2 \mathrm{~h}$ glucose levels, VAT, BMI or $\mathrm{HbA}_{1 \mathrm{c}}$. Analysis further found no associations between total pancreatic fat, intralobular fat or parenchymal fat with insulin secretion or beta cell function in NGT participants: total insulin secretion (all $p>0.7$ ), adaptation index (all $p>0.6$ ) and insulinogenic

Fig. 4 Association of total pancreatic fat, intralobular fat and parenchymal fat with $(\mathbf{a}-\mathbf{c})$ insulin AUC 120 min, $(\mathbf{d}-\mathbf{f})$ adaptation index (OGIS $\times$

$\mathrm{AUC}_{\mathrm{CP}}$ ), and (g-i) insulinogenic index in participants with NGT. Data adjusted for age, sex and OGIS
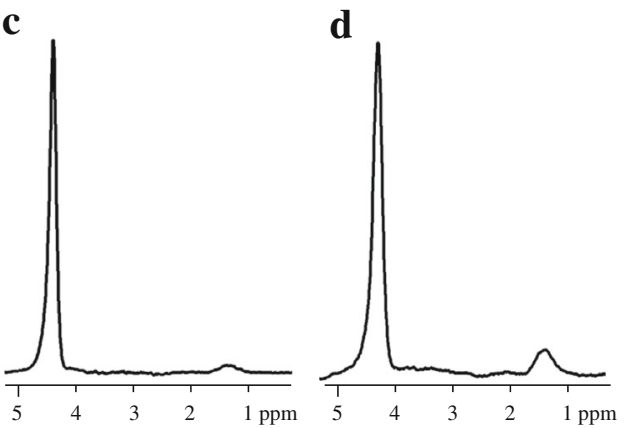

broad peak at $1.3 \mathrm{ppm}$; (c) $5 \%$ intralobular pancreatic fat with low amplitude broad peak at $1.3 \mathrm{ppm}$; (d) $11 \%$ pancreatic fat intentionally visceral-fat-doped voxel

index (all $p>0.2$ ) (Fig. 4). Furthermore, no associations were found between any non-invasive pancreatic fat measurements and beta cell function in IFG/IGT participants - total insulin secretion (all $p>0.4$ ), adaptation index (all $p>0.3$ ), insulinogenic index (all $p>0.5$ ) - or patients with type 2 diabetes - total insulin secretion (all $p>0.5$ ), adaptation index (all $p>0.4$ ) and insulinogenic index (all $p>0.3$ ) (Fig. 5).

\section{Discussion}

This study found that pancreatic fat deposition in the region of the pancreas displayed an inhomogeneous distribution of adipose tissue infiltration that increased with decreasing glucose

b

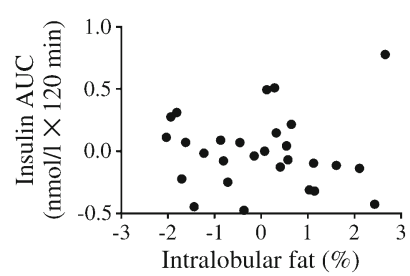

e
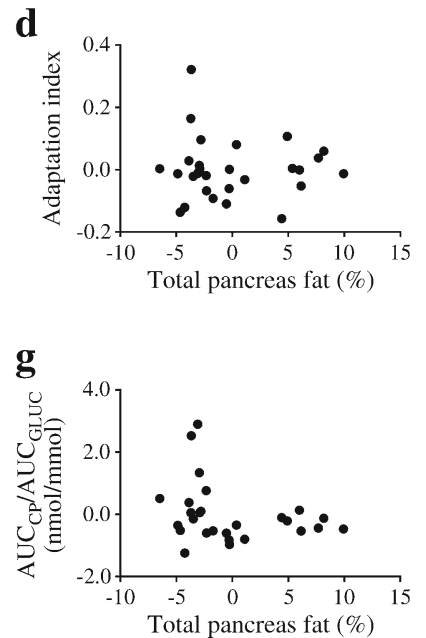

a
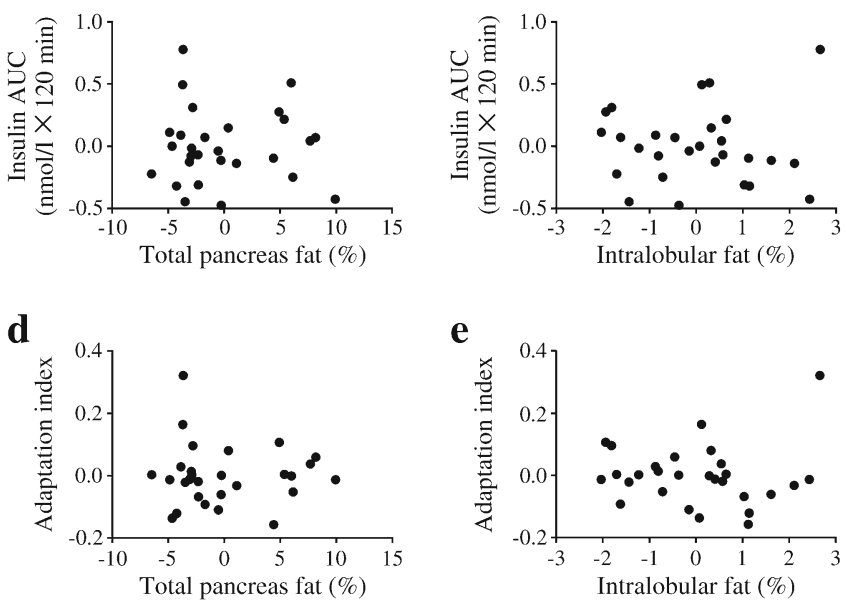

h

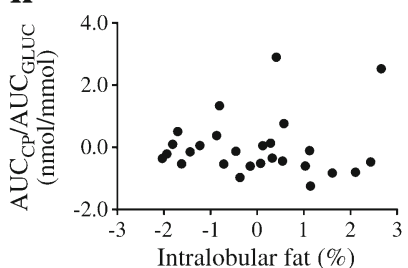

c

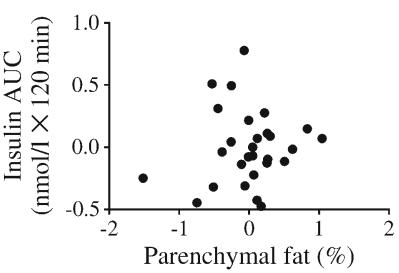

f

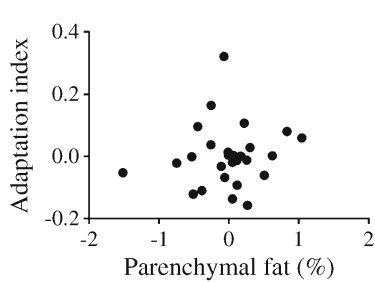

i

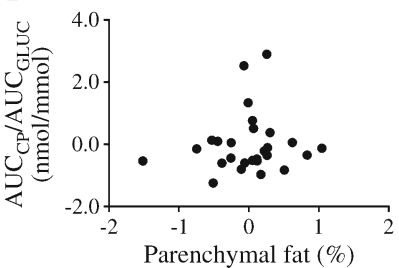


Fig. 5 Individual analysis of the association of total pancreatic fat, intralobular fat and parenchymal fat with $(\mathbf{a}-\mathbf{c})$ insulin AUC $120 \mathrm{~min},(\mathbf{d}-\mathbf{f})$ adaptation index $\left(\mathrm{OGIS} \times \mathrm{AUC}_{\mathrm{CP}}\right)$, and $(\mathbf{g}-\mathbf{i})$ insulinogenic index within participants with IFG/IGT (squares) and type 2 diabetes (triangles). Data adjusted for age, sex and OGIS $\mathbf{a}$

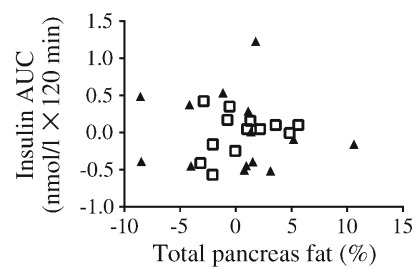

d

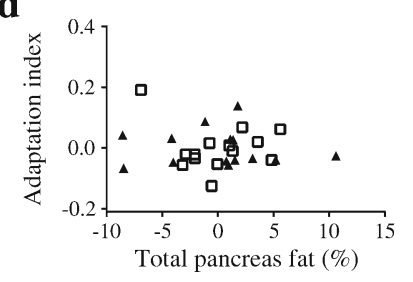

g

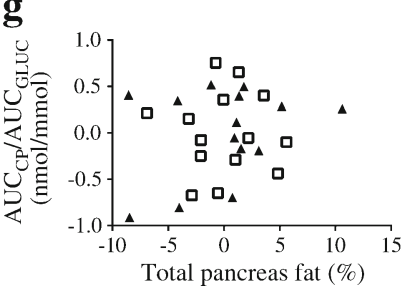

b

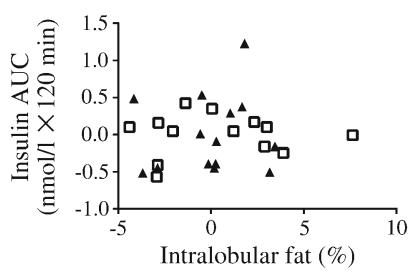

e

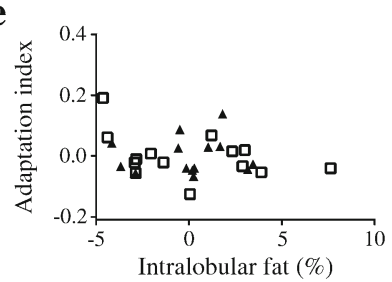

h

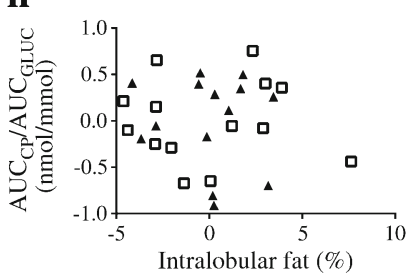

c

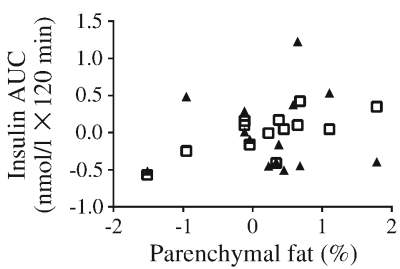

f
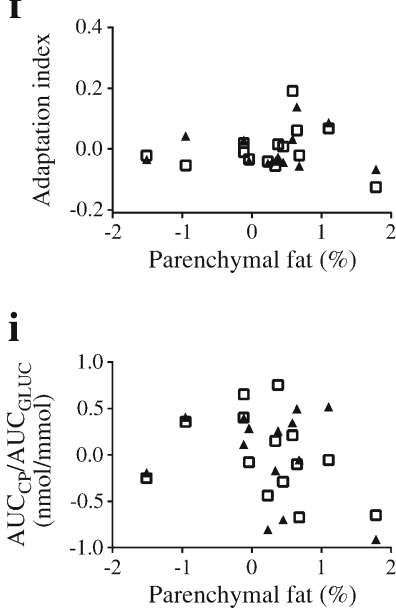

tolerance. On the other hand, MRI pancreatic fat measurements revealed large regions of parenchymal tissue void of relevant lipid accumulation in all participants, providing evidence against uniform pancreatic steatosis. Interestingly, despite positive correlation with age, no measure of pancreatic fat was associated with any variable of beta cell function, regardless of glucose tolerance status.

Values obtained from standard ${ }^{1} \mathrm{H}$-MRS pancreatic fat measurements were in line with previous reports [17, 24], and were highest in patients with type 2 diabetes [17, 19, 24]. It has been hypothesised through studies in humans [12, $17,20]$ and Zucker fatty rats $[13,26,40]$ that ${ }^{1} \mathrm{H}$-MRS measurements can therefore serve as a non-invasive biomarker of pancreatic steatosis, and a surrogate for triacylglycerol accumulation in beta cells. However, the present comparison of ${ }^{1} \mathrm{H}$-MRS techniques shows that non-invasive pancreatic fat measurements with localization via clinical $\mathrm{T} 2$-weighted images are not an accurate description of whole-organ fat, but are influenced by the inhomogeneous distribution of peripancreatic or interlobular fat that was visible in the highresolution mDIXON fat/water images [28]. While this inhomogeneous distribution of fat is in contrast to previous MRI reports $[16,18]$, histological analyses of human pancreas tissue $[11,15,41,42]$ confirm the inhomogeneous distribution of pancreatic adipose tissue infiltration found in this study. This inhomogeneous distribution of adipose tissue could also explain the discrepancies in ${ }^{1} \mathrm{H}-\mathrm{MRS}$ [17, 19, 24] and MRI $[16,18,21,38]$ pancreatic fat studies, and suggests that noninvasive methods that assess whole-organ adipose tissue

infiltration $[14,43]$ might provide more insight into the association between pancreas adipose tissue infiltration and beta cell function.

Analysis of all ${ }^{1} \mathrm{H}-\mathrm{MRS}$ fat spectra also revealed that total pancreatic fat and intralobular fat measurements were representative of lipid accumulation within adipose tissue, rather than parenchymal pancreatic triacylglycerol accumulation. Triacylglycerol overflow in the liver results in a distribution of narrow fat peaks representing different fatty acid chains, such as methyl (0.9 ppm) and methylene (1.3 ppm) [7, 39], whereas ${ }^{1} \mathrm{H}$-MRS pancreatic fat measurements revealed a single broad methylene peak even at high fat concentrations. This broad fat peak suggests that the fat is in a different chemical environment than the intracellular fat typically found in hepatocytes and myocytes [44], and thus experiences a different spin-spin relaxation. This hypothesis was verified by an in vivo pancreatic fat measurement intentionally doped with VAT; the results of the VAT-laden voxel matched a typical ${ }^{1} \mathrm{H}$ MRS pancreatic fat spectrum (Fig. 3).

Parenchymal pancreatic fat measurements further disprove the hypothesis of a uniform pancreatic steatosis [27] by showing, in all individuals, regions of parenchymal pancreatic tissue void of lipid accumulation. The fat-fraction algorithm within low-fat PRIDE reduced noise bias [45] at low-fat fractions, thereby improving the accuracy of parenchymal fat measurements [29, 38], but with an underestimation of results below $2 \%$. Previous MRI methods used for pancreatic fat measurements either did not distinguish between parenchymal tissue and adipose tissue infiltration [18] or were hindered by 
low signal-to-noise ratio [21] and thick slices [16, 22]. Thus, results could also have been limited by the accuracy of the MRI method $[16,38,46]$ for measuring low fat values or the method was not able to capture the irregular shape of the pancreas; higher pancreatic fat values could have reflected VAT infiltration. These findings are verified by histological reports of human pancreatic tissue that have clearly identified ectopic pancreatic fat as adipocyte infiltration visible between exocrine cells [11, 15, 41, 42]. Additionally, whole-organ autopsies have reported that pancreatic fat consists only of patchy areas of intralobular or perilobular fat [15], or pancreatic fat dissecting islands of pancreatic tissue [41]; to date, no human autopsy has reported evidence of intracellular pancreatic fat and beta cell dysfunction caused by pancreatic steatosis $[11,15,42]$. Taken together, the present data provide evidence that the accumulation of triacylglycerols within pancreatic parenchymal tissue is not a major contributor to the development of type 2 diabetes.

This study also confirmed reports that pancreatic adipose tissue infiltration is positively associated with age [14, 15, 24]. However, while adipose tissue infiltration increased with decreasing glucose tolerance, no associations were found with fasting or $2 \mathrm{~h}$ glucose, BMI, HCL or VAT. To date, the factors that lead to pancreatic adipose tissue infiltration are unknown as the associations of pancreas adipose tissue infiltration with BMI [18, 19], VAT [18, 21, 24] or HCL [19, 21, 24] are inconclusive. Previously, it has been reported that pancreatic fat infiltration is negatively associated with beta cell function in non-diabetic men [19] and prediabetic IFG/IGT patients [18], but not in patients with type 2 diabetes [19]. On the contrary, in this study we were unable to find any association between pancreatic adipose tissue infiltration and beta cell function regardless of glucose tolerance status. However, it has also been reported that pancreatic adipose tissue infiltration increases with age and BMI, but not type 2 diabetes [47]. Correspondingly, even participants with type 2 diabetes in this study had pancreases void of adipose tissue infiltration, and increased intralobular fat was found only in older participants with type 2 diabetes. Thus, this study also supports the hypothesis that adipose tissue rather reflects the replacement for parenchymal tissue loss $[15,48]$ that has been shown to occur with age [14] or type 2 diabetes [49], and only serves as a marker $[24,50]$ of beta cell dysfunction after the loss of parenchymal volume and corresponding loss of beta cell mass [49].

Among the limitations of this study was the rather small number of participants included in the study. Also, the older age of participants and patients with type 2 diabetes might have contributed to higher pancreatic fat values. Additionally, parenchymal fat measurements were not capable of detecting fat concentrations below $2 \%$ [29], or the accumulation of fat directly within islets. Last, the route of glucose administration in the OGTT compared with the intravenous glucose tolerance test could affect the assessment of beta cell function. However, the OGTT-derived beta cell variables have been validated against those obtained from intravenous glucose loading [36] and also more closely resemble physiological conditions of insulin secretion.

In conclusion, this study found that pancreatic fat consists of an inhomogeneous distribution of adipose tissue infiltration rather than uniform pancreatic steatosis. Adipose tissue infiltration increased with age and decreasing glucose tolerance. However, no associations were found with beta cell function regardless of glucose tolerance status. Taken together, the pancreas does not appear to be another target organ for abnormal endocrine function due to ectopic parenchymal fat storage.

Acknowledgements The authors would like to thank: A. Nagel and N. Achterath for patient care and maintaining the MRI; K. Tinnes and M. Esser for participant recruitment; and U. Wilke of Philips Healthcare for assistance within the Philips Achieva platform. Some of the data were previously presented as an abstract at the 74th Scientific Sessions of the American Diabetes Association in 2014.

Funding This work was supported by the Ministry of Science and Research of the State of North Rhine-Westphalia (MIWF NRW) and the German Federal Ministry of Health (BMG). This study was also supported in part by grants from the Federal Ministry for Research (BMBF) to the German Center for Diabetes Research (DZD), the Helmholtz Alliance with Universities (Imaging and Curing Environmental Metabolic Diseases; ICEMED), the German Research Foundation (DFG; SFB 1116, B05) and the Schmutzler Stiftung, Germany.

Duality of interest The funding bodies and Philips Healthcare had no role in the study design, data collection or analysis, or the decision to publish the manuscript. The authors declare that there is no duality of interest associated with this manuscript.

Contribution statement PB planned the study, carried out the MRS measurements and analysis, and wrote the manuscript. CK and GP wrote the manuscript and researched data. JB, BN, PN, KW, KM and JS researched data and reviewed/edited the manuscript. KS conducted the statistical analysis, and reviewed the manuscript. GP carried out the analysis of the OGTT results and JB provided the LF-PRIDE software package. MR planned the study, wrote the manuscript, contributed to the discussion and reviewed/edited the manuscript. All authors provided final approval for the publication of this work. MR is the guarantor of this work, has full access to all the data in the study and takes responsibility for the integrity of the data and accuracy of analysis.

\section{References}

1. Butler AE, Janson J, Bonner-Weir S, Ritzel R, Rizza RA, Butler PC (2003) Beta-cell deficit and increased beta-cell apoptosis in humans with type 2 diabetes. Diabetes 52:102-110

2. Kahn SE, Hull RL, Utzschneider KM (2006) Mechanisms linking obesity to insulin resistance and type 2 diabetes. Nature 444:840-846

3. Federici M, Hribal M, Perego L et al (2001) High glucose causes apoptosis in cultured human pancreatic islets of Langerhans: a potential role for regulation of specific Bcl family genes toward an apoptotic cell death program. Diabetes 50:1290-1301 
4. Unger RH, Grundy S (1985) Hyperglycaemia as an inducer as well as a consequence of impaired islet cell function and insulin resistance: implications for the management of diabetes. Diabetologia 28:119121

5. Robertson RP, Harmon J, Tran PO, Poitout V (2004) Beta-cell glucose toxicity, lipotoxicity, and chronic oxidative stress in type 2 diabetes. Diabetes 53(Suppl 1):S119-S124

6. Prentki M, Nolan CJ (2006) Islet beta cell failure in type 2 diabetes. J Clin Invest 116:1802-1812

7. Krssak M, Hofer H, Wrba F et al (2010) Non-invasive assessment of hepatic fat accumulation in chronic hepatitis $\mathrm{C}$ by $1 \mathrm{H}$ magnetic resonance spectroscopy. Eur J Radiol 74:e60-e66

8. Perseghin G, Scifo P, De Cobelli F et al (1999) Intramyocellular triglyceride content is a determinant of in vivo insulin resistance in humans: a 1H-13C nuclear magnetic resonance spectroscopy assessment in offspring of type 2 diabetic parents. Diabetes 48:1600-1606

9. Krssak M, Falk Petersen K, Dresner A et al (1999) Intramyocellular lipid concentrations are correlated with insulin sensitivity in humans: a 1H NMR spectroscopy study. Diabetologia 42:113-116

10. Szczepaniak LS, Babcock EE, Schick F et al (1999) Measurement of intracellular triglyceride stores by $\mathrm{H}$ spectroscopy: validation in vivo. Am J Physiol 276:E977-E989

11. Pinnick KE, Collins SC, Londos C, Gauguier D, Clark A, Fielding BA (2008) Pancreatic ectopic fat is characterized by adipocyte infiltration and altered lipid composition. Obesity (Silver Spring) 16:522530

12. Lee Y, Lingvay I, Szczepaniak LS, Ravazzola M, Orci L, Unger RH (2010) Pancreatic steatosis: harbinger of type 2 diabetes in obese rodents. Int J Obes (Lond) 34:396-400

13. Lee Y, Hirose H, Ohneda M, Johnson JH, McGarry JD, Unger RH (1994) Beta-cell lipotoxicity in the pathogenesis of non-insulindependent diabetes mellitus of obese rats: impairment in adipocytebeta-cell relationships. Proc Natl Acad Sci U S A 91:10878-10882

14. Saisho Y, Butler AE, Meier JJ et al (2007) Pancreas volumes in humans from birth to age one hundred taking into account sex, obesity, and presence of type-2 diabetes. Clin Anat 20:933-942

15. Stamm BH (1984) Incidence and diagnostic significance of minor pathologic changes in the adult pancreas at autopsy: a systematic study of 112 autopsies in patients without known pancreatic disease. Hum Pathol 15:677-683

16. Patel NS, Peterson MR, Brenner DA, Heba E, Sirlin C, Loomba R (2013) Association between novel MRI-estimated pancreatic fat and liver histology-determined steatosis and fibrosis in non-alcoholic fatty liver disease. Aliment Pharmacol Ther 37:630-639

17. Lingvay I, Esser V, Legendre JL et al (2009) Noninvasive quantification of pancreatic fat in humans. J Clin Endocrinol Metab 94:4070-4076

18. Heni M, Machann J, Staiger $\mathrm{H}$ et al (2010) Pancreatic fat is negatively associated with insulin secretion in individuals with impaired fasting glucose and/or impaired glucose tolerance: a nuclear magnetic resonance study. Diabetes Metab Res Rev 26:200-205

19. Tushuizen ME, Bunck MC, Pouwels PJ (2007) Pancreatic fat content and beta-cell function in men with and without type 2 diabetes. Diabetes Care 30:2916-2921

20. Szczepaniak LS, Victor RG, Mathur R et al (2012) Pancreatic steatosis and its relationship to beta-cell dysfunction in humans: racial and ethnic variations. Diabetes Care 35:2377-2383

21. Le KA, Ventura EE, Fisher JQ et al (2011) Ethnic differences in pancreatic fat accumulation and its relationship with other fat depots and inflammatory markers. Diabetes Care 34:485-490

22. Schwenzer NF, Machann J, Martirosian P et al (2008) Quantification of pancreatic lipomatosis and liver steatosis by MRI: comparison of in/opposed-phase and spectral-spatial excitation techniques. Investig Radiol 43:330-337

23. Hu HH, Kim HW, Nayak KS, Goran MI (2010) Comparison of fatwater MRI and single-voxel MRS in the assessment of hepatic and pancreatic fat fractions in humans. Obesity (Silver Spring) 18:841847

24. van der Zijl NJ, Goossens GH, Moors CC et al (2011) Ectopic fat storage in the pancreas, liver, and abdominal fat depots: impact on beta-cell function in individuals with impaired glucose metabolism. J Clin Endocrinol Metab 96:459-467

25. Sakuraba H, Mizukami H, Yagihashi N, Wada R, Hanyu C, Yagihashi S (2002) Reduced beta-cell mass and expression of oxidative stress-related DNA damage in the islet of Japanese Type II diabetic patients. Diabetologia 45:85-96

26. Shimabukuro M, Higa M, Zhou YT, Wang MY, Newgard CB, Unger RH (1998) Lipoapoptosis in beta-cells of obese prediabetic fa/fa rats. Role of serine palmitoyltransferase overexpression. J Biol Chem 273: 32487-32490

27. Smits MM, van Geenen EJ (2011) The clinical significance of pancreatic steatosis. Nat Rev Gastroenterol Hepatol 8:169-177

28. Eggers H, Brendel B, Duijndam A, Herigault G (2011) Dual-echo Dixon imaging with flexible choice of echo times. Magn Reson Med 65:96-107

29. Begovatz P, Koliaki C, Szendroedi J, Giani G, Bunke J, Roden M (2014) Pancreas fat via dual-echo mDIXON imaging shows that intracellular fat does not accumulate within the pancreas of healthy and T2DM subjects. Proc Intl Soc Magn Reson 23:3608 (Abstract)

30. Pacini G, Mari A (2003) Methods for clinical assessment of insulin sensitivity and beta-cell function. Best Pract Res Clin Endocrinol Metab 17:305-322

31. Szendroedi J, Anderwald C, Krssak M et al (2009) Effects of highdose simvastatin therapy on glucose metabolism and ectopic lipid deposition in nonobese type 2 diabetic patients. Diabetes Care 32: 209-214

32. Mari A, Pacini G, Murphy E, Ludvik B, Nolan JJ (2001) A modelbased method for assessing insulin sensitivity from the oral glucose tolerance test. Diabetes Care 24:539-548

33. Ahren B, Pacini G (2004) Importance of quantifying insulin secretion in relation to insulin sensitivity to accurately assess beta cell function in clinical studies. Eur J Endocrinol 150: 97-104

34. Kautzky-Willer A, Krssak M, Winzer C et al (2003) Increased intramyocellular lipid concentration identifies impaired glucose metabolism in women with previous gestational diabetes. Diabetes 52: 244-251

35. Utzschneider KM, Prigeon RL, Faulenbach MV et al (2009) Oral disposition index predicts the development of future diabetes above and beyond fasting and 2-h glucose levels. Diabetes Care 32: 335-341

36. Tura A, Kautzky-Willer A, Pacini G (2006) Insulinogenic indices from insulin and C-peptide: comparison of beta-cell function from OGTT and IVGTT. Diabetes Res Clin Pract 72:298-301

37. Utzschneider KM, Prigeon RL, Tong J et al (2007) Within-subject variability of measures of beta cell function derived from a $2 \mathrm{~h}$ OGTT: implications for research studies. Diabetologia 50: $2516-2525$

38. Livingstone RS, Begovatz P, Kahl S, et al. (2014) Initial clinical application of modified Dixon with flexible echo times: hepatic and pancreatic fat assessments in comparison with ${ }^{1} \mathrm{H}$ MRS. MAGMA 27:397-405

39. Hamilton G, Yokoo T, Bydder M et al (2011) In vivo characterization of the liver fat ${ }^{1} \mathrm{H}$ MR spectrum. NMR Biomed 24:784-790

40. Shimabukuro M, Zhou YT, Levi M, Unger RH (1998) Fatty acidinduced beta cell apoptosis: a link between obesity and diabetes. Proc Natl Acad Sci U S A 95:2498-2502

41. Nghiem DD, Olson PR, Ormond D (2004) The "fatty pancreas allograft": anatomopathologic findings and clinical experience. Transplant Proc 36:1045-1047 
42. Navina S, Acharya C, DeLany JP et al (2011) Lipotoxicity causes multisystem organ failure and exacerbates acute pancreatitis in obesity. Sci Transl Med 3:107ra110

43. Lim S, Bae JH, Chun EJ et al (2014) Differences in pancreatic volume, fat content, and fat density measured by multidetector-row computed tomography according to the duration of diabetes. Acta Diabetol 51:739-748

44. Szczepaniak LS, Dobbins RL, Metzger GJ et al (2003) Myocardial triglycerides and systolic function in humans: in vivo evaluation by localized proton spectroscopy and cardiac imaging. Magn Reson Med 49:417-423

45. Reeder SB, Cruite I, Hamilton G, Sirlin CB (2011) Quantitative assessment of liver fat with magnetic resonance imaging and spectroscopy. J Magn Reson Imaging 34:729-749
46. Sijens PE, Edens MA, Bakker SJ, Stolk RP (2010) MRI-determined fat content of human liver, pancreas and kidney. World $\mathrm{J}$ Gastroenterol 16:1993-1998

47. Saisho Y, Butler AE, Butler PC (2008) Pancreatic fat content and beta-cell function in men with and without type 2 diabetes: response to Tushuizen et al. Diabetes Care 31:e38, author reply e39

48. Walters MN (1966) Adipose atrophy of the exocrine pancreas. J Pathol Bacteriol 92:547-557

49. Rahier J, Guiot Y, Goebbels RM, Sempoux C, Henquin JC (2008) Pancreatic beta-cell mass in European subjects with type 2 diabetes. Diabetes Obes Metab 10(Suppl 4):32-42

50. van Raalte DH, van der Zijl NJ, Diamant M (2010) Pancreatic steatosis in humans: cause or marker of lipotoxicity? Curr Opin Clin Nutr Metab Care 13:478-485 